Cipango
Cipango

$21 \mid 2014$

Nouveaux regards sur les arts de la scène japonais II

BARDY Yannick, Sanctuaires shintô et sociétés locales dans le Japon de l'époque d'Edo : l'exemple de la province d'Izumi

thèse de doctorat, sous la direction de François MACÉ, Inalco, 2013, $425+65 \mathrm{p}$.

\title{
OpenEdition
}

Journals

Édition électronique

URL : https://journals.openedition.org/cipango/2379

DOI : $10.4000 /$ cipango.2379

ISSN : 2260-7706

Éditeur

INALCO

Édition imprimée

Date de publication : 31 décembre 2014

ISSN : 1164-5857

Référence électronique

"BARDY Yannick, Sanctuaires shintô et sociétés locales dans le Japon de l'époque d'Edo : l'exemple de la province d'Izumi », Cipango [En ligne], 21 | 2014, mis en ligne le 07 septembre 2016, consulté le 30 juin 2021. URL : http://journals.openedition.org/cipango/2379; DOI : https://doi.org/10.4000/cipango. 2379

\section{(2) $(1) \Theta$}

Cipango est mis à disposition selon les termes de la Licence Creative Commons Attribution - Pas d'Utilisation Commerciale 4.0 International. 
BARDY Yannick, Sanctuaires shintô et sociétés locales dans le Japon de l'époque d'Edo : l'exemple de la province d'Izumi, thèse de doctorat, sous la direction de François MACÉ, Inalco, 2013, 425+65 p.

Depuis une trentaine d'années, certaines des connaissances sur l'époque d'Edo (1600-1868) issues de l'histoire marxiste de l'après-guerre font l'objet de remises en cause par certains courants de l'historiographie japonaise récente. C'est le cas des études portant sur les rapports entre la religion et la société. En effet, jusqu'aux années 1980-1990, les historiens tendaient à considérer la vie religieuse comme un élément de faible incidence pour la compréhension de cette époque et se contentaient de travaux sur des grands établissements religieux, sur des questions d'ordre institutionnel ou encore sur des événements populaires de masse. Durant cette période, ce sont principalement les ethnologues et folkloristes qui ont interrogé les pratiques religieuses populaires. D'autres remises en question viennent des travaux sur les statuts sociaux et leurs marges qui réinterrogent l'organisation de la société au-delà des principes politiques ou des idées des penseurs de l'époque. Questionnant la réalité des organisations sociales en province au travers d'exemples précis de couples sanctuaire shintô-villageois, notre thèse s' inscrit dans la continuité de ces deux courants historiographiques.

Nous avons choisi d'étudier les cas de cinq ensembles de villages de la province d'Izumi, au sud d'Osaka. Les sanctuaires étudiés ne sont pas de grandes institutions religieuses mais de petits sanctuaires de province, proches de la vie de tous les jours. De plus, ils sont liés à des groupes de villages constitués autour d'eux et qui, à ce titre, existent en dehors des cadres administratifs officiels. Ces sanctuaires ne comptent donc pas parmi les plus nombreux mais sont d'un niveau qui impose une organisation importante de la part des villageois. Aussi, leur étude permet de faire ressortir de manière précise les pratiques socioreligieuses et les modes organisationnels des villageois pour la gestion de ces sanctuaires. Dans le même temps, nous nous sommes attachés à mettre en lumière les différents types de religieux, 
tant bouddhistes que shintô, chargés de l'exécution des rites et de l'entretien quotidien des sanctuaires, ainsi que les groupes s'efforçant de les organiser, tels que les organisations de prêtres des familles de cour Yoshida ou Shirakawa. Ces cinq cas sont étudiés au cours de quatre parties dont les trois premières présentent des ensembles de villages situés au centre de la province, dans le territoire de l'actuelle municipalité d' Izumi.

Dans notre première partie, il s'agit tout d'abord d'étudier les sept villages de la vallée Ikeda, gérant en commun un sanctuaire Kasuga. Dans le premier chapitre, nous avons mis en évidence le mode de fonctionnement du système de gestion, ouvert puisque chaque villageois en fait partie, et qui est à ce titre bien loin du système fermé des miyaza qui ont fait l'objet de tant d'études ethnologiques ou folkloriques. Cette organisation est dirigée par un petit groupe composé du prêtre et d'officiers villageois représentant chaque village. Nous y évoquons également le rôle des groupes de jeunes des villages.

Dans le second chapitre, nous nous sommes penchés sur la prêtrise et l'évolution du mode de désignation du prêtre, d'une prêtrise tournant au sein des branches d'une même famille, à sa captation par l'une de ces branches et sa transformation en charge héréditaire avec l'appui des officiers villageois et de la maison Yoshida. C'est l'occasion d'étudier l'implication de cette maison auprès de ce sanctuaire de province, ainsi que la concurrence entre prêtres shintô et desservants bouddhiques (moine shasō) au début du XIX ${ }^{\mathrm{e}}$ siècle.

Enfin, sachant que ces desservants résident tous deux dans un autre village que celui où se situe le sanctuaire, à plus de cinq kilomètres, nous avons poussé notre travail jusqu'à l'étude de leur village de résidence dans le troisième chapitre. Cela nous a permis de faire ressortir la place particulière de la famille du prêtre, ses liens anciens de patronage avec le monastère du shasō, ainsi que des évolutions profondes datant de la fin du XVIIII ${ }^{\mathrm{e}}$ siècle dues au développement de la marchandisation dans cette période, notamment l'apparition d'une nouvelle couche de notables liée au commerce de l'huile.

La seconde partie, qui se réferre aux travaux de Mita Satoko, est consacrée à l'étude d'une autre communauté de villages (le pays de Shinoda), organisée elle aussi selon un système ouvert pour gérer le sanctuaire Hijiri (ou Shinoda) et son territoire, ainsi que les monts Shinoda. Cette communauté est composée de sept villages qui y participent pleinement et de deux autres dont les droits sont limités: 
Ma.i, un petit village de danseurs, et Minami-ōji, un village de parias dont la population au début du XIX ${ }^{\mathrm{e}}$ siècle représentait plus de la moitié de la somme des habitants du total des neuf villages.

Après un premier chapitre qui décrit ces villages, le sanctuaire et l'emprise très importante des premiers sur le second et sur son personnel religieux (prêtres et moines), le second chapitre s'attache à étudier les évolutions au sein du sanctuaire autour des années 1755-1762. Ces changements sont avant tout relatifs à l'organisation hiérarchique des desservants et à la distribution des rôles religieux entre eux, au travers de leurs affiliations, l'une à l'organisation de prêtres de la maison Shirakawa, l'autre au Ninna-ji. Cela pose notamment la question du rôle des problèmes locaux dans le développement des organisations des Yoshida et des Shirakawa. Or ces changements entraînent des contestations suivies de procès en hauts lieux qui vont modifier la hiérarchie originelle. Désormais, les villageois ne peuvent plus gérer les territoires du sanctuaire comme ils l'entendent et doivent tenir des registres de comptes précis qu'ils présentent chaque année aux desservants.

Le troisième chapitre est consacré à une dispute qui a secoué ces villages autour de l'année 1828. L'origine de cette querelle était la demande des villageois d'affilier les territoires du sanctuaire Hijiri au Ninna-ji, dans un but en apparence religieuse. Or, en réalité, cet objectif en cachait deux autres : obtenir une exemption d'impôts sur les terres du sanctuaire cultivées par des villageois, et écarter de ces dernières les parias de Minami-ōji. Ces deux objectifs font ressortir des problèmes et notamment l'opposition d'une certaine élite locale autour de l'évolution de l'organisation administrative de la seigneurie des Hitotsubashi, branche collatérale de la famille du shogun, à laquelle appartiennent ces villages. Ils révèlent également une situation conflictuelle entre certains de ces villages et les parias, dont la cause n'est peut-être pas tant dans le statut des parias que dans leur nombre qui les amène à exploiter des terres dans les villages voisins et autour du sanctuaire.

La troisième partie est consacrée à l'étude de deux sanctuaires d'une vallée plus à l'intérieur des terres : la vallée Yokoyama. Les deux premiers chapitres portent sur la partie dite « haute » de cette vallée, qui consiste en réalité en deux villages liés à un sanctuaire Kaminomiya. Nous nous appuyons sur les travaux de Yamashita Sōichi pour montrer la progressivité du découpage en villages au début du XVII ${ }^{e}$ siècle. La question de l'établissement et la modification progressive de la 
fonction de shōya fait ressortir l'importance d'une première famille de notables locaux. Cette charge qui portait à l'origine sur l'ensemble de la vallée s'est ensuite fixée au seul village de Butsunami, mettant en évidence non seulement la progressivité du découpage administratif des anciennes communautés médiévales mais aussi des découpages internes des nouveaux villages, bien loin d'être des ensembles homogènes.

Puis, nous abordons d'abord la question de la prêtrise du sanctuaire et de la manière dont elle a été attachée à une autre famille de notables, puis le fonctionnement de l'assemblée du sanctuaire (miyaza), organisation limitée à quelques lignées familiales des deux villages, portant des noms particuliers uniquement en usage dans le cadre des assemblées. Ce point permet de faire ressortir les luttes d'influences entre villages et entre les deux maisons de notables (shöya de Butsunami et prêtre). Enfin, au travers de l'étude de l'organisation au sein des deux villages d'un rite commun à trente-sept villages de la province, le Dainenbutsu, nous revenons sur le rôle et les luttes des deux familles de notables ainsi que sur les tentatives de contrôle de l'assemblée du sanctuaire à partir du XVIII ${ }^{\mathrm{e}}$ siècle.

Le troisième chapitre est consacré à l'étude de la partie « basse » de la vallée et de son sanctuaire Shimonomiya. Dans un premier temps nous avons étudié la composition du miyaza en deux assemblées, elles-mêmes formées à partir de celles de trois villages pour l'une, quatre pour l'autre. Ces assemblées sont elles aussi limitées à quelques lignées qui portent des noms en usage dans le cadre des rites du miyaza. Or certains de ces noms sont communs avec ceux de l'assemblée du Kaminomiya. Ce point, ainsi que les liens mythiques des divinités et les danses communes aux deux sanctuaires suggèrent une proximité ancienne entre les deux établissements et leurs communautés. Cette partie permet également d'étudier les diverses charges au sein de l'assemblée, telles que celles de prêtre, dévolues à une famille de notables. Dans un second temps, l'étude des rites bouddhiques fait ressortir le rôle important de l'un des villages dont le shōya conserve les sūtras, ainsi que celui du système de patronage des monastères par des familles locales, et leur soutien par le réseau de parenté et de voisinage de ces familles. Enfin, l'affirmation de la primauté du prêtre en matière religieuse apparaît clairement au milieu du XVIII ${ }^{\mathrm{e}}$ siècle.

La quatrième partie est consacrée au sanctuaire Ōiseki du sud de la province et au groupe de quatre villages qui le supporte. Les deux premiers chapitres sont 
consacrés à l'organisation du sanctuaire, aux positions respectives des moines shasō et du prêtre, ainsi qu'à l'existence d'une famille de notables conjuguant la charge de toshiyori à celle de jinin, l' « homme du dieu », en apparence religieuse mais qui semble tenir plutôt du patronage dans ce cas-ci. Ce point est l'occasion d'interroger les rapports entre l'organisation de la maison Yoshida avec les prêtres de province, au milieu du XVII ${ }^{\mathrm{e}}$ siècle d'abord, puis dans la première moitié du $\mathrm{XIX}^{\mathrm{e}}$ siècle. Dans le troisième chapitre, après avoir analysé le mode de gestion du sanctuaire par les villageois, nous nous sommes intéressés aux gains, mais également aux pertes considérables que la gestion et la protection des territoires du sanctuaire ont entraînées pour les villageois, et des évolutions socio-économiques qui y sont liées. Nous y abordons également la querelle liée à la prêtrise, à l'issue de laquelle le prêtre gagne en indépendance grâce à l'intervention de la maison Yoshida, et qui use de cette position nouvelle pour commencer à contester au moine et aux villageois l'usage des territoires situés dans l'enceinte du sanctuaire.

Grâce à ce travail s'approchant de l'histoire locale à la japonaise et des case-studies ou de la microhistoire, nous avons pu étudier ces couples villageois-sanctuaire de la manière la plus exhaustive possible puisque, outre l'évocation du syncrétisme shintô-bouddhique ou la diversité des statuts des religieux et l'évolution de leur organisation, nous avons aussi emprunté à l'histoire sociale et à l'histoire économique pour interroger les liens entre des villages pourtant séparés sur le plan administratif mais liés par leur rapport avec le sanctuaire commun. Nous avons également pu étudier la participation de laïcs au culte, la place des parias dans certains de ces groupes de villages, ou encore le fonctionnement institutionnel des villages et leur évolution socio-économique.

Notre thèse fait notamment apparaître la diversité des sanctuaires et de leurs organisations de fidèles. Nous avons mis en évidence le rôle des notables dans le soutien à ces sanctuaires et aux groupes les gérant, mais également la manière dont ils se servent de leur position au sein desdits groupes pour conserver leur place dans la société, avec parfois des effets pervers pouvant entrainer leur appauvrissement. Nous avons également confirmé qu'un sanctuaire de province ne peut s'envisager sans les fidèles et les villageois qui les supportent et organisent les rites. Enfin, ceux que nous avons étudiés dans notre thèse existent en dehors des cadres officiels fixés au cours du XVII ${ }^{\mathrm{e}}$ siècle et perdurent jusqu'à l'ère Meiji. Or ces groupes de villages s'organisant autour d'un sanctuaire de pays semblent liés aux anciennes 
472 Cahiers d'études japonaises n²1

communes médiévales, et leur étude laisse apparaître des liens avec des organisations sociales préexistantes, telles que des notables issus de l'ancienne couche de petits seigneurs qui s'appuient sur les éléments subsistants de cette époque pour essayer de maintenir et améliorer leur position au sein de la société.

Malgré cela, des évolutions sont évidentes, notamment à partir du milieu du $\mathrm{XVIII}^{\mathrm{e}}$ siècle, autour de l'émergence d'une catégorie de prêtres shintô se professionnalisant ou tout au moins s'affiliant aux organisations des Yoshida ou des Shirakawa et gagnant ainsi en influence, tant par les appuis qu'ils y trouvent que par les savoirs religieux qui leurs sont transmis. Bien qu'étant un exercice local, l'étude du contexte des affiliations notamment permet de mettre en évidence une évolution dans ces deux maisons qui passent d'une évidente passivité, à une grande fébrilité sans doute liée à la concurrence à laquelle elles se livrent à la fin du shogunat. 